\title{
Customize Linux Operating System and Adding Own Feature
}

\author{
Madhuri \\ Bhaganagare \\ B.E. (CSE) \\ SKNSCOE, \\ Pandharpur \\ Pandharpur 413304
}

\author{
Pradnya \\ Ghongade \\ B.E. (CSE) \\ SKNSCOE, \\ Pandharpur \\ Pandharpur 413304
}

\author{
Pooja Jadhav \\ B.E. (CSE) \\ SKNSCOE, \\ Pandharpur \\ Pandharpur 41330
}

\author{
Snehal Nakate \\ B.E. (CSE) \\ SKNSCOE, \\ Pandharpur \\ Pandharpur 413304
}

\begin{abstract}
A Linux distribution is an operating system made as a collection of software based around the Linux kernel and often around a package management system. A typical Linux distribution comprises a Linux kernel, GNU tools and libraries, additional software, documentation, a window system, window manager, and a desktop environment. Most of the included software is free software/open-source software which is distributed by its maintainers both as compiled binaries and in source code form, allowing users to modify and compile the original source code if they wish. Other software included with some distributions may be proprietary and may not be available in source code form. Linux distributions are almost universally Unix-like as described; the most notable exception is Android, which does not include a command-line interface and programs made for typical Linux distributions. There are currently over six hundred Linux distributions; over three hundred of those are in active development, constantly being revised and improved. One can distinguish between commercially backed distributions, such as Fedora (Red Hat), opens USE (SUSE), Ubuntu (Canonical Ltd.) and Mandriva Linux (Mandriva)[1], and entirely community-driven distributions, such as Debian, Slack ware, Gentoo[1] and Arch Linux.
\end{abstract}

There is no facility such as Task Manager in all Linux distributions. Virtual Task Manager is the new feature we are going to add in our Customized operating system. Also we are going to provide a user friendly GUI which can be easily handled by new user. Ubuntu has the built-in utility to monitor or kill system running processes which acts like the "Task Manager", it's called System Monitor. Ctrl+Alt+Del shortcut key by default is used to bring up the log-out dialog on Ubuntu Unity Desktop. It is not useful for users who are used to quick access to the Task Manager. To change the settings of the key, open Keyboard utility from the Unity Dash (or System Settings -> Keyboard). On Shortcuts tab -> Custom Shortcuts, click the plus icon to add a shortcut. type in name Task Manager and command gnome-system-monitor.

\section{Keywords}

Operating system, Task Manager, Linux.

\section{INTRODUCTION}

UNIX as the world known it today is the happy outcome of the proverbial rags-to-riches story. What is now heralded as the most powerful and popular multiuser Operating System(OS) had a very humble beginning in the austere premises of AT and T's Bell laboratories[2], the fertile spawning ground of many a land mark in computer history.
The UNIX operating system was born in the late 1960s. It originally began as a one man project led by Ken Thompson of Bell Labs, and has since grown to become the most widely used operating system. In the time since UNIX was first developed, it has gone through many different generations and even mutations. Some differ substantially from the original version, like Berkeley Software Distribution (BSD) or Linux. Others still contain major portions that are based on the original source code. Linux distributions are primarily based on free and open-source software, at least partially; that part includes the Linux kernel and usually a very large collection of software of all sorts.[4]

\section{LITERATURE REVIEW}

Open Source: (source code is readily available and free to modify)[3] FreeBSD - no access currently available at UMBC. Linux Distributions - access is available in the form of dual-boot PCs scattered throughout the Engineering building. There are also several Linux servers (linux.gl.umbc.edu) through which access are available.

Red Hat (used by UMBC) and the Fedora Project (maintained by Red Hat)

Debian

Ubuntu

SuSE[3]

Slackware

And many others..

\section{SCOPE FOR IMPLEMENTATION}

A task manager is a system monitor program used to provide information about the processes and programs running on a computer, as well as the general status of the computer. Some implementations can also be used to terminate processes and programs, as well as change the processes priority. In some environments, users can access a task manager by pressing the buttons Control-Alt-Delete. Task managers can display currently running services (processes) as well as those that were stopped. They can display information about the services (such as Process ID and group) if known. In Windows you can easily kill any task by pressing Ctrl+Alt+Del[6] and bringing up the task manager. Linux running the GNOME desktop environment[5] (i.e. Debian, Ubuntu, Linux Mint, etc.) has a similar tool that can be enabled to run exactly the same way. 


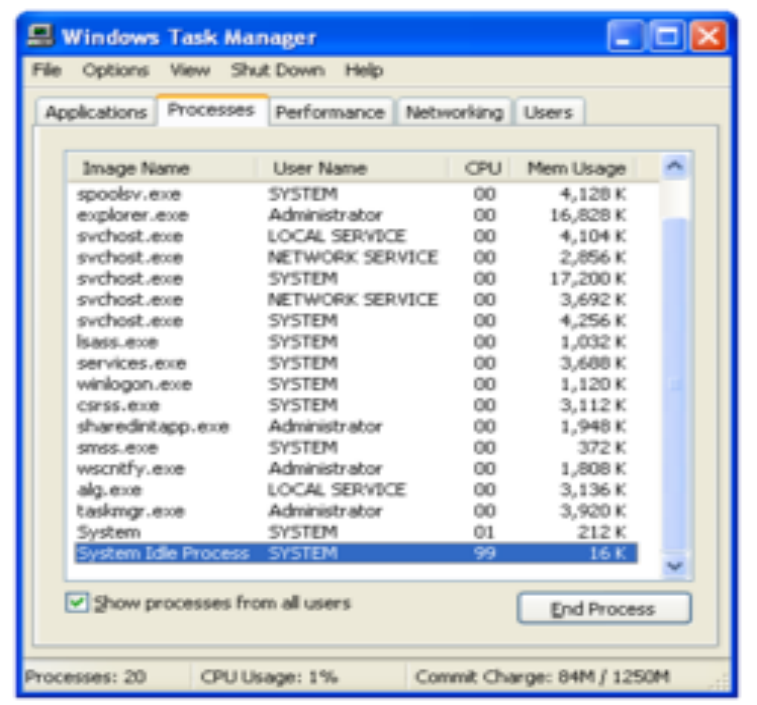

Fig 1: Task manager in Windows Operating system

\section{ACKNOWLEDGMENT}

We would like to express my deep sense of gratitude to my Guide Prof. B. M. Kore, Prof. A. A. Rajguru, HOD, ComputerScience and Engineering Department, for their invaluable help And guidance for the duration of project. We are highly indebted To him for constantly encouraging us by giving critics on our Work. We are grateful to him for providing us the support and Confidence.

\section{REFERENCES}

[1] http://en.wikipedia.org/wiki/List_of_Linux_distributions

[2] http://en.wikipedia.org/wiki/Ubuntu_\%28

[3] http://en.wikipedia.org/wiki/SUSE_LinuxLinuxdistributi on $\% 29$

[4] http://en.wikipedia.org/wiki/Yellow_Dog_Linux

[5] http://en.wikipedia.org/wiki/Linux

[6] http://www.computerhope.com/jargon/t/taskmana.htm 